\title{
Statistical Model Selection Procedure in Data Modeling
}

\author{
Ritei Shibata \\ Department of Mathematics, \\ Keio University, 3-14-1 \\ Hiyoshi Kohoku, Yokohama, \\ 223-8522, Japan \\ E-mail: shibata@math.keio.ac.jp
}

\section{Data Modeling}

Statistical model selection is a key to the success of statistical modeling for exploring something new in science, controlling a system or making decision in every day's life. However, a lot of things have to be done before applying a formal model selection procedure. First of all, data should be collected carefully and organized properly. Data cleaning is often indispensable. A part of data is often missing, or a kind of calibration is necessary. Improper treatment of data in this stage may destroy the meaning of the model selection which comes later.

Seeking for an appropriate statistical model is time consuming and a hard job, but spending enough time with data for exploring possible models is rewarding. This is a key to the success of data modeling. It is dangerous to confine yourself to a particular model which had been established. If you confine yourself to such a model, you may miss a big chance of finding better models or take a risk of applying an erroneous model. Look at data carefully and imagine what kind of mechanism is hidden behind the data. Several ideas for the modeling may come out. Try and check the goodness of fit of each model you imagine by your eyes or by simple statistics. Always think about the meaning of each model. If there is any doubt, through away such a model even if it shows a good fit to the data.

A model selection procedure using a specific criterion, like $\mathrm{AIC}, \mathrm{BIC}, \mathrm{MDL}$ or $\mathrm{ABIC}$ almost comes at the end of data modeling. In other words, it is useful to employ such a criterion only when all candidate models are meaningful and there is no good reason for discriminating them. Automatic application of such a criterion at an early stage of data modeling is most dangerous. You may miss an important feature of the mechanism hidden behind the data. In this paper, we will illustrate a full path of statistical modeling procedure of underlying system. As an example of modeling, we take commodity futures prices. However the approach is not restricted to such an economic data but rather common for any data unless the structure is well known beforehand.

\section{Financial Data}

Financial market is a large complicated open system. One of well known approaches of the modeling of financial data is through a stochastic process like Martingale or Stochastic Differential Equation driven by a Brownian motion. However, it captures only a part of the features of financial trading, a very stable part or the equilibrium ( for example see [1]). To model its dynamics, data driven modeling is most desirable. One of such approaches can be found in Shibata and Miura[2]. They dealt with seven different daily interest rate series. A seven variate AR model, that is, a linear system, is fitted to the residual time series after two deterministic movements, long term and short term trends, are removed. The criterion $\mathrm{AIC}$ is used only at the last stage of selecting the order of the AR model. The order selected so as to minimize AIC is 2 and the AR model shows a quite good fit. We could not have such a simple model without decomposing each original series into two deterministic components and one stochastic component.

\section{$3 \quad$ Futures Price Modeling}

An example of futures price in Japan is given in Figure 1. There are six different daily gold futures prices (yen/gram) at a time $t$ due to lagged maturities, that is, 2 month, 4 month, 6 month, 8 month, 10 month and 12 month ahead maturities. Each futures price series runs over a year.

Most well known model for futures price ( for example, see Hull [3]) is

$$
F_{t, T}=S_{t} \exp \left(\int_{t}^{T} r_{s} d s\right)
$$

where $F_{t, T}$ is the futures price at time $t$, which matures at time $T, S_{t}$ is the current price and $r_{t}$ is the interest rate at time $t$. Therefore, it is natural to begin with checking if this model is valid for actual futures price data. Fortunately in case of gold a reliable current price series is available, which is called LOCO- 
Futures prices of gold

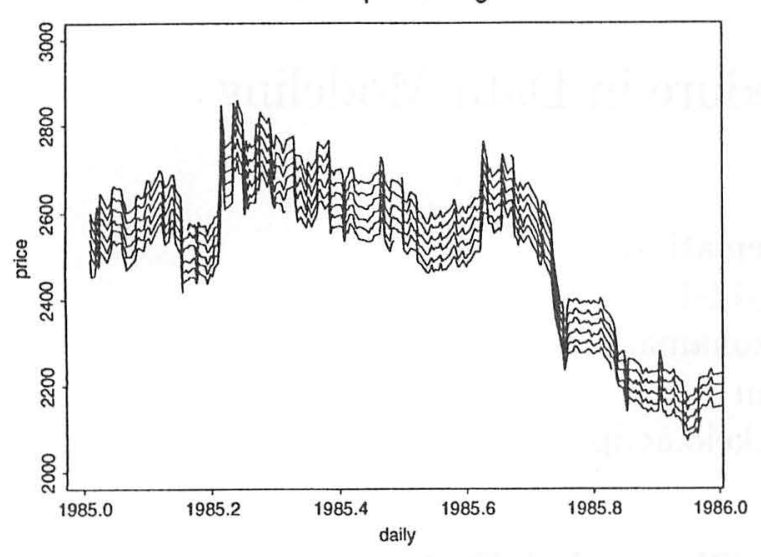

Figure 1: Futures Prices of Gold for 350 days in the Beginning of 1985 .

TOKYO-GOLD price. A simple regression model

$$
\log \frac{F_{t, T}}{S_{t}}=r(T-t)+\epsilon_{t}
$$

is fitted but it does not yield any reasonable estimate of the interest rate. It is improved if a weighted least squares estimation procedure is applied in conjunction with the amount of dealings. The estimated interest rate behaves very similarity to the official discount rate. However significant nonlinearity remains in the residuals [4]. The limitations of such a data modeling are now clear.

1. It is not applicable to other futures unless reliable current price is available. As far as we know, no such price is available except gold.

2. Estimated interest rate is an average over a year.

3. Interest rates inferred from different series show significant inconsistency.

\subsection{Stochastic Component and Non- stochastic Component}

After playing with data, we found that the following model explains well the behavior of futures prices. The idea is that we can cancel out the current price $S_{t}$ by taking the $\log$ ratio,

$$
Z_{t}^{(k)}=\frac{12}{k} \log \frac{F_{t, T+30 k}}{F_{t, T}}
$$

This ratio is expected to behave similarly to an averaged interest rate over $k$ months as far as the model (1) is valid. In case of gold, we can create five different time series $Z_{t}^{(k)}, k=2,4,6,8,10$ over whole time period by switching the series into a new futures price series which just starts after the maturity. The top panel in Figure 2 shows an example $Z_{t}^{(2)}$ with $k=2$. The time period

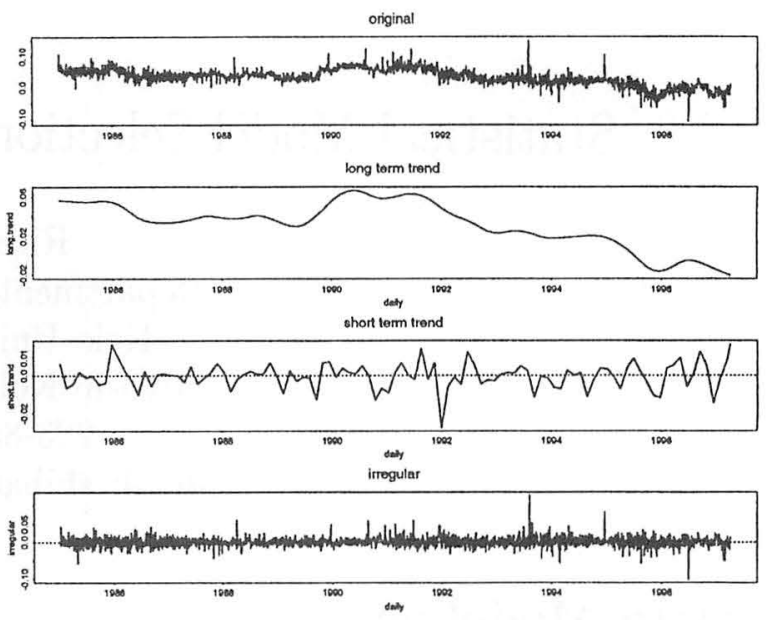

Figure 2: Decomposition of $Z_{t}^{(2)}$.

is from the 4th of January, 1985 to the 31st of March, 1997.

However this series behaves differently from the interest rate. To extract the effect of changes of interest rate, a smoothing technique, local regression algorithm lowess which is used in modeling the seven interest rate series in Shibata and Miura[2] is applied. The long term trend, which varies in a yearly span, extracted from $Z_{t}^{(2)}$ is shown on the second panel. By applying the same technique, we can extract the short term trend which varies in a monthly span from the residuals of the long term trend. It is shown on the 3rd panel. The final residual series is shown on the last panel.

Now $Z_{t}^{(2)}$ is decomposed into the long term trend, the short term trend and the residuals. We regard only the residuals as stochastic and others as exogenous effect to the futures market from outside of the market. Introduction of such a distinction between stochastic and non stochastic components makes modeling simpler. Since interest rate is exogenous, the long term trend should have a close connection to the interest rate.

\subsubsection{Non-stochastic Component}

In Figure 3, the five solid lines show the long term trends of $Z_{t}^{(k)}, k=2,4,6,8,10$, that is, interest rates inferred from gold futures prices, the dotted lines show three a part of Euro Yen interest rates with different maturities such as 3 month, 6 month and 12 month as a reference. The chain line which behaves stepwise shows the official discount rate. From this figure, we see that the interest rates inferred from gold futures, which behave closely each other, behave closely to the market interest rates before the bubble economy (which starts from the end of 1989 ) and then behave closely to the official discount rate. It is quite natural that the interest rate inferred from gold futures reflects the market interest rate in normal economy but stays relatively 




Figure 3: Interest rates inferred from gold futures(solid lines), Interest rates in market (dotted lines) and Official discount rate (chain line).

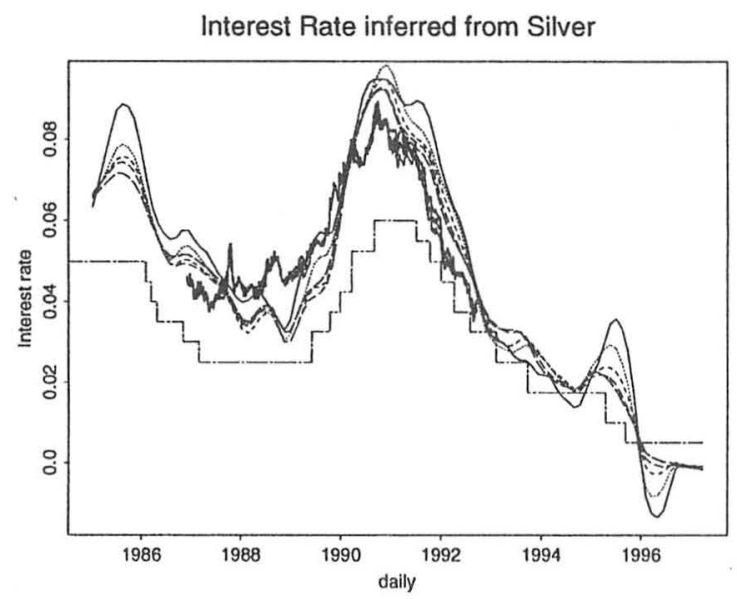

Figure 4: Interest rates inferred from silver futures(solid lines), Interest rates in market (dotted lines) and Official discount rate (chain line).

stable when economy bubbles up. It is also interesting to note that the behavior in the last several years after 1995. The official discount rate is almost zero because of heavy recession of Japanese economy but which can not be negative. However the inferred interest rate is honest and reflect negative effective interest rate.

Figure 4 is a similar plot for silver futures. The difference from gold is clear. The inferred interest rates behave not so closely each other as gold but closely to the market interest rates. This behavior is natural because gold is highly convertible to cash but silver is not. Main use of silver is for industry, so that the future prices directly reflect the market interest rate.

The short term trends show almost the same cyclic behavior. It is still open for future research to explain the real meaning of the behavior.

\subsection{Stochastic Component}

For futures price of gold or silver, we have five different residual series as is seen in the previous section. It is worth to analyze them as a multiple time series $W(t)$ by combining them into a vector. We may then find a stochastic relationship among those series. A simple model to $W(t)$ is an AR model

$W(t)+\Phi_{1} W(t-1)+\Phi_{2} W(t-2)+\cdots+\Phi_{p} W(t-p)=\xi(t)$.

The minimum AIC principle suggests the choice of order $p=1$ both for gold and silver. However this is only an omnibus choice of the order. Appropriate order may vary according to the combination of variates of $W(t)$ and $W(t-1)$. We searched for such choices not only for the case $p=1$ but for $p=2$ and we have a very simple model for $W(t)$ both for gold and silver,

$$
W_{i}(t)+\phi_{i} W_{i}(t-1)=\xi_{i}(t), i=1, \cdots, 5 .
$$

The coefficient $\phi_{i}$ remain almost the same value 0.5 . This result shows that stochastic component of the relative movement of futures has a simple structure, that is, it depends on the past only through the last value of itself.

\section{Concluding Remarks}

As has been illustrated in the previous sections, to find a most appropriate model is a series of explorations and validations. Automatic application of an information criterion may result in totally unreliable model. Even when applying an information criterion almost at the last stage of modeling, it is not good idea to confine ourselves to small differences of the values of the criterion. Meaningfulness of the model is always at first priority as far as the residuals of the fit show no peculiar pattern.

\section{References}

[1] Longstaff, F. and Schwartz, E. Interest Rate Volatility and the Term Structure: A Two-Factor General Equilibrium Model, Journal of Finance, XLVII, pp. 1259-1282, 1992.

[2] Shibata, R. and Miura R. Decomposition of Japanese Yen Interest Rate Data Through Local Regression, Financial Engineering and the Japanese Markets, 4, 125-146, 1997.

[3] Hull, J. Introduction to Futures and Options Markets, Prentice Hall, 1991.

[4] Miyazawa, M. and Shibata, R. Data Modeling Commodity Futures, presented in Japan Statistical Society Meeting, July, 1999. 
